\title{
The role of occludin in vascular barrier function in vivo: do we need to re- examine?
}

\author{
Ruyuan Zhang, Yiyun Liu, Dechang Chen ${ }^{*}$ and Yaoqing Tang ${ }^{*}$
}

Dear editor,

Septic patients often undergo altered mental status. One possible reason for this brain dysfunction is thought to be related to vascular leakage caused by systemic inflammation. The recent article by Erikson et al. [1] showed that major tight junctions protein occludin in brain autopsy specimens in sepsis was downregulated and that the downregulation of occludin was related with severe organ dysfunction and systemic inflammation. While these findings are novel and objective, we feel it may be necessary to interpret the data with caution regarding to equating the decrease of occludin expression with blood-brain barrier dysfunction in this and other related research.

Since its discovery by Shoichiro Tsukita, a pioneer in the field of tight junction, most of the data in literature about the causal role of occludin in barrier function are from in vitro experiments. However, occludin-deficient mice do not display a perturbation of barrier function, with a complex pathophysiological and inexplicable phenotype [2] which even disappointed its discoverer Shoichiro Tsukita. Moreover, few reports regarding the in vivo barrier function of occludin mainly focused on epithelial cells [3]. Surprisingly, research from Charles M. Rice's lab showed that occludin acts as a hepatitis $C$ virus entry factor [4] and thus won the 2016 Albert Lasker Award. In another recent report dedicated to the memory of Dr. Shoichiro Tsukita, occludin deficiency in mice causes deafness while not affecting the tight junction structure or barrier [5].

* Correspondence: chendechangsh@hotmail.com;

yaoqing.tang@hotmail.com

Department of Critical Care Medicine, Rui Jin Hospital, Shanghai Jiao Tong University School of Medicine, Shanghai 200025, China
Therefore, due to the lack of vascular barrier disruption in occludin-deficient mice, additional in vivo animal experiments may be needed to make the conclusions for those studying the role of occludin in vascular barrier function in vivo and those using occludin expression as an indicator of blood-brain barrier function more reliable. However, there is a possibility that occludin ${ }^{-1-}$ mice may be able to compensate for occludin loss. It also cannot be excluded that the level of occludin plays limited roles under normal physiological conditions but modulates the sensitivity of blood vessels to pathophysiologically relevant stressors. Some questions that need to be answered in the future include the following: Will postnatal endothelial-specific occludin knockdown in mouse brain lead to vascular leak? Are occludindeficient mice more sensitive to sepsis? Will endothelial-specific occludin overexpression prevent or limit sepsis-induced brain vascular leak in vivo?

\section{Acknowledgements}

None.

\section{Authors' contributions}

RZ designed the paper. All authors participated in drafting and reviewing. All authors read and approved the final version of the manuscript.

\section{Funding}

This work was supported by the National Natural Science Foundation of China (NSFC, Grant No. 81772041).

\section{Availability of data and materials Not applicable.}

Ethics approval and consent to participate Not applicable.

Consent for publication Not applicable. 


\section{Competing interests}

The authors declare that they have no competing interests.

Received: 7 July 2020 Accepted: 14 July 2020

Published online: 22 July 2020

\section{References}

1. Erikson K, Tuominen H, Vakkala M, Liisanantti JH, Karttunen T, Syrjala H, AlaKokko TI. Brain tight junction protein expression in sepsis in an autopsy series. Crit Care. 2020;24:385.

2. Saitou M, Furuse M, Sasaki H, Schulzke JD, Fromm M, Takano H, Noda T, Tsukita S. Complex phenotype of mice lacking occludin, a component of tight junction strands. Mol Biol Cell. 2000;11:4131-42.

3. Marchiando AM, Shen L, Graham WV, Weber CR, Schwarz BT, Austin JR 2nd, Raleigh DR, Guan Y, Watson AJ, Montrose MH, Turner JR. Caveolin-1-

dependent occludin endocytosis is required for TNF-induced tight junction regulation in vivo. J Cell Biol. 2010;189:111-26.

4. Ploss A, Evans MJ, Gaysinskaya VA, Panis M, You H, de Jong YP, Rice CM. Human occludin is a hepatitis $C$ virus entry factor required for infection of mouse cells. Nature. 2009;457:882-6.

5. Kitajiri S, Katsuno T, Sasaki H, Ito J, Furuse M, Tsukita S. Deafness in occludindeficient mice with dislocation of tricellulin and progressive apoptosis of the hair cells. Biol Open. 2014;3:759-66.

\section{Publisher's Note}

Springer Nature remains neutral with regard to jurisdictional claims in published maps and institutional affiliations. 